\title{
Experimental CubeSat-based Network for Alert Messages Broadcasting
}

\author{
Alessio Fanfani ${ }^{1}$, Simone Morosi ${ }^{2}$, Luca Simone Ronga ${ }^{3}$, \\ Sara Jayousi ${ }^{2}$, Enrico Del $\operatorname{Re}^{2}$ and Luca Rossettini ${ }^{1}$ \\ ${ }^{1}$ D-Orbit Srl, Via G. Mazzini 2, Milano, 20123, Italy \\ ${ }^{2}$ Department of Information Engineering of the University of Florence, \\ Via S. Marta 3, Florence, 50139, Italy \\ ${ }^{3}$ CNIT Research Unit of the University of Florence, Via S. Marta 3, \\ Florence, 50139, Italy \\ E-mail: alessio.fanfani@deorbitaldevices.com; \\ luca.rossettini@deorbitaldevices.com; simone.morosi@unifi.it; \\ sara.jayousi@cnit.it; enrico.delre@unifi.it; luca.ronga@ieee.org
}

Received 10 February 2017; Accepted 31 May 2017;

Publication 19 February 2018

\begin{abstract}
In this paper an end-to-end proof of concept of a low complexity CubeSatbased network architecture for alert dissemination is presented. The adoption of a CubeSat for alert messages broadcasting, of MAMES (Multiple Alert Message Encapsulation over Satellite) as alert messages transport protocol and of a satellite Portable SDR (Software Defined Radio) terminal as the receiver represent the key elements of the proposed architecture. The proposed system is described, highlighting the functioning of the whole "alert chain" from the generation to the reception of MAMES messages: particularly, the experimental activities carried out to prove the feasibility of the proposed alert network and the applicability of MAMES to LEO satellites are reported.
\end{abstract}

Keywords: Alerting system, Alert Messaging, Nano satellite, CubeSat, Software Defined Radio, Web Services, Multiple Alert Message Encapsulation protocol over Satellite (MAMES).

Journal of Mobile Multimedia, Vol. 14_1, 1-24.

doi: 10.13052/jmm1550-4646.1411

This is an Open Access publication. (c) 2018 the Author(s). All rights reserved. 


\section{A. Fanfani et al.}

\section{Introduction}

Recent technologies have now opened the doors for new satellite applications and services. Thanks to innovative manufacturing processes (e.g. additive manufacturing) the miniaturization of electronics components has allowed the origin of a new class of satellite, called "Small-Sat", that is growing exponentially in last years. The term "Small-Sat" was initially used for mini and micro satellites with mass lower than $500 \mathrm{~kg}$; however, today it often refers to Nano and Pico satellites or even smaller in size [1].

The growing trend of Small-Sat is confirmed by global experts in satellite market consulting and analysis: Euroconsult says that a total of 551 small satellites has been launched over the past 5 years and 1380 are expected to be launched up to 2020 [2]. A quantitative description of the exponential growing trend of the number of small satellites launched per year is reported in Figure 1 [1]. The most common form factor for the small satellites is the CubeSat standard that consists of a cube with edges of length $10 \mathrm{~cm}$ and a mass of up to $1.33 \mathrm{~kg}$. This standard unit has been defined to be a 1U CubeSat and can be combined in more complex structures in order to obtain bigger CubeSat platform as $2 \mathrm{U}, 3 \mathrm{U}, 6 \mathrm{U}$ and $12 \mathrm{U}$. The standardization of CubeSat satellites has allowed a global viral diffusion of them: several launch opportunities with traditional and new generation of launchers are rising all over the world,

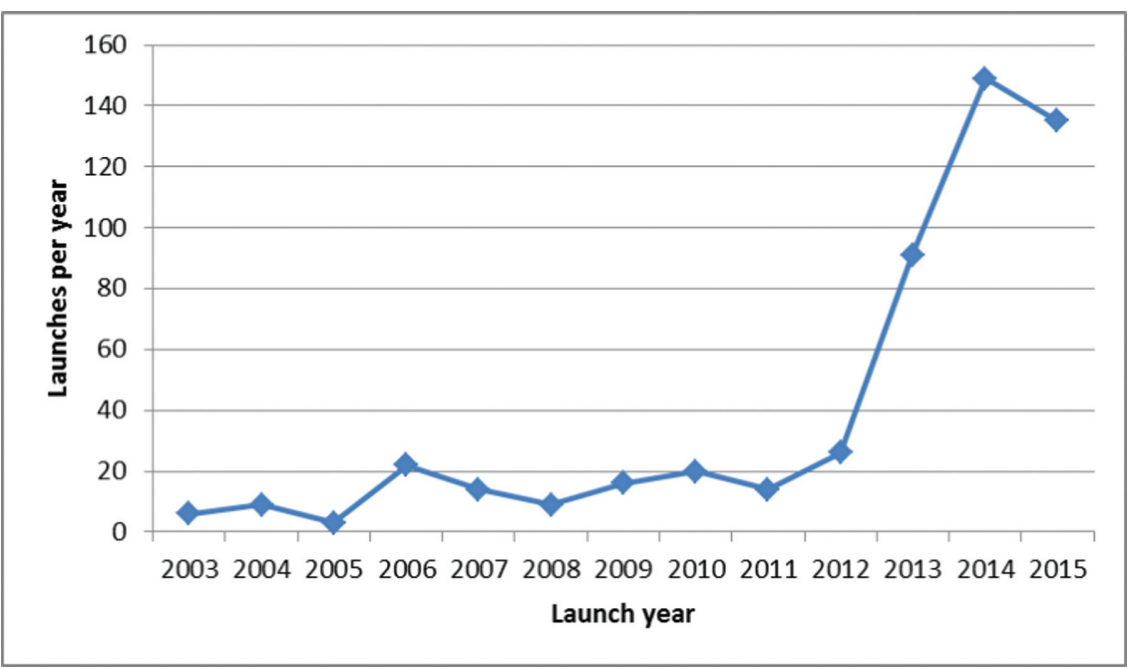

Figure 1 Number of small satellites launched per year [1]. 
hundreds of companies and universities are developing satellite sub-systems based on COTS electronics and the satellite cost and manufacturing time have been reduced respectively up to few dozen of thousand dollars and a couple of weeks [3]. The reduction of both the manufacturing and the launch costs as well the brief manufacturing time will benefit the diffusion of satellite constellation. In particular, Small Satellites will generate new opportunities for the communication services and the Earth observation application that are based on huge number of satellites in Low Earth Orbit (LEO). Adam Keith, Managing Director of Euroconsult Canada, said that "Earth observation is expected to exhibit the strongest growth in terms of launched units; over 2,100 satellites are anticipated from 2016 to 2025. Four companies plan to launch more than 1,400 satellites during this period alone: Planet, Spire, BlackSky Global and Satellogic. Satellite communication smallsats will also increase significantly to nearly 800 during the same period of time. There are six constellations foreseen, however OneWeb makes up the bulk of these units" [4].

The diffusion of CubeSat Constellations for communication services has been slowed down by the limited power capability of these nano-platforms. Anyway, few ambitious communication services have been provided also with $1 \mathrm{U}$ CubeSat platform. An example is the the AAUSAT3 mission which is carried out by Aalborg University (Denmark) for validating an SDR automatic identification system (AIS) signals receiver for cubesat. The AIS is an automatic tracking system that is used for maritime traffic monitoring based on low-rate digital data exchange over VHF signals. The satellite AAUSAT3 was launched on February 2013 and has received more than 9000 AIS messages on daily basis [5]. Another interesting project is Outernet that aims to broadcast around the world free content retrieved from the web through satellites, making information effectively available for all people [6]. Outernet will launch their three $1 \mathrm{U}$ nanosatellites late in 2017. Finally the exponential global growth of IoT business is attracting also satellite service providers: an example is Astrocast Project that is envisaged to deploy a network of $3 \mathrm{U}$ cubesats in Low-Earth Polar Orbit for offering global Machine to Machine (M2M) services like as remote monitoring, geolocalization, intelligent data collection and predictive maintenance [7].

The increasing population of satellites leads also to a consequent growing of space debris that currently includes more than 300 million of fragments and about 5,000 of thousands defunct satellites. In order to guarantee a sustainable access to space, it is necessary to stop the systematic increment of nonoperative satellites in orbit [8]. This seems to be feasible thanks to preventive 
debris removal solution, as the Decommissioning Device developed by D-Orbit, whereby a dedicated and independent propulsion system is installed on the satellite prior to launch allowing a safe and quick decommissioning of the spacecraft before it will become a debris [9].

Another enabling factor for the diffusion of next generation satellite networks is the evolution of Software Defined Radios (SDR) [10], which are devices composed by a wide-band RF front-end plus a direct modulator/demodulator and a Digital-Analog/Analog-Digital Converters (DAC/ADC) [11]. Digital data, that can be acquired at several sampling rates and central frequencies, are elaborated by a software running on a microprocessor that performs all typical radio's functions. Usually, the software code is generated by GNURadio [12], a user-friendly software development environment. Thanks to SDR, all functionalities of an expensive satellite transceiver and decoder can be implemented in a single board that includes a computer.

The use of the previous mentioned technologies allows the definition of low complexity systems which can be adopted in a large variety of application contexts. Focusing on emergency management scenarios, a growing number of standardization activities and research projects addresses the topic of Emergency Services provided by satellite systems [13-15] and the exploitation of different communication channels (e.g. Global Navigation Satellite System [16]). The provision of an effective alerting service relies on a timely and reliable distribution of alert messages to the intended audience of the incident affected area. Already existing alerting systems/technologies and an efficient transport of different alert message formats are key elements in the provisioning of an alerting service. To this aim an extensible multiple alert message encapsulation protocol for transporting differently formatted alert messages over satellite links has been defined by ETSI: MAMES (Multiple Alert Message Encapsulation over Satellite) [17, 18].

This paper proposes a low complexity satellite alert network based on the adoption of: i) a CubeSat for alert messages broadcasting; ii) MAMES protocol for alert messages transport; and iii) SDR technology for messages reception. The experimental activities are carried out in the framework of the D-SAT Mission (Section 2) and aim at demonstrating the feasibility of the proposed low complexity alert network and the MAMES applicability to LEO satellites. The paper is organized as follows: Section 2 presents the operative context and objectives of D-SAT mission. An overview of the overall architecture of the proposed alerting system and a detailed description of its main components, including the MAMES Protocol, the Space (CubeSat) 
and Ground (Satellite Mission Control Center and portable SDR user terminal) Segments, is reported in Section 3. Section 4 is devoted to the description of the results and current status of the experimental activities. Finally, conclusions are drawn in Section 5.

\section{Operative Context: DSAT Mission}

The proposed satellite alert network will be experimented in the framework of the D-SAT Mission. D-SAT spacecraft has been designed and manufactured by D-Orbit (Italy) and it will be launched by the Polar Satellite Launch Vehicle (PSLV) in LEO Sun-Synchronous Orbit at $500 \mathrm{~km}$ altitude in June 2017. The Mission includes two main experimental activities: the SatAlert Experiment and the Decommissioning Device Experiment.

\subsection{SatAlert Experiment - Objectives and Details}

The SatAlert experiment aims to verify the transmission setup of MAMES messages from the MAMES Alert Provider to the MAMES Alert Receiver (see Section 3). The ground infrastructure responsible for the MAMES Message generation and the transmission over the space segment, the on-board software that parses and broadcasts MAMES messages and the portable SDR UT for the reception of the alert will be validated.

During the visibility period, the Satellite Earth station sends to the satellite a MAMES message (maximum size of $1.5 \mathrm{kB}$ ) that will be processed by the satellite and stored in the On-Board Computer memory for broadcasting upon reception of a proper trigger command sent by the Earth station (Figure 2).

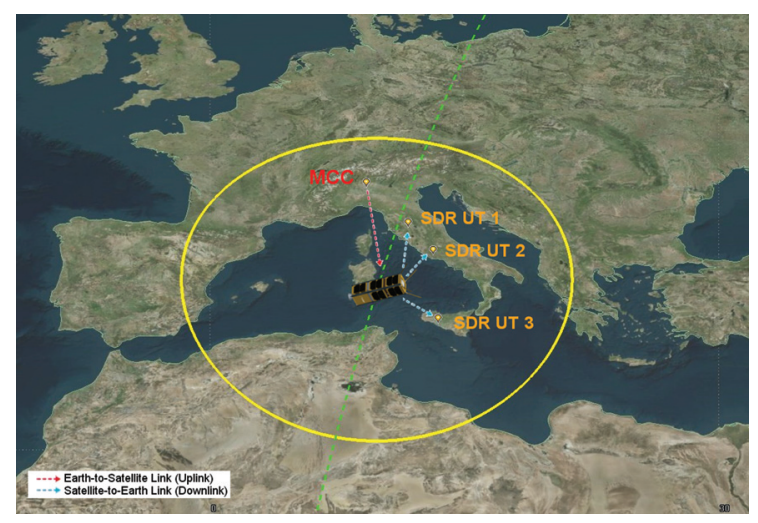

Figure 2 D-SAT ground footprint. 
According to the international agreements on non-interference regulated by no. 4.4 of the ITU Radio Regulations, MAMES broadcast transmission will be activated only during the available visibility windows in order not to cause harmful interference to other satellite and terrestrial services. The yellow circle in Figure 2 shows the satellite footprint on ground that covers a wide area on ground comprising several nations. For each user on ground, 3 or 4 visibility windows/day (depending on the specific day) and a visibility period of about $480 \mathrm{~s}$ will be provided.

\subsection{Decommissioning Device Experiment - Objectives and Details}

D-SAT Mission will validate the functioning of D-Orbit Decommissioning device in space through the active satellite disposal at the end of mission. Thus, D-SAT will be the first CubeSat ever to be actively de-orbited in a safe and reliable way. After two months of nominal operations and experimental validation activities of the MAMES Protocol, the D-Orbit Decommissioning Device will be activated and D-SAT will be removed from its operational orbit.

\section{System Overview: Low Complexity Satellite Alert Network}

The overall system architecture developed for the demonstration of a low complexity satellite alert network for MAMES messages broadcasting is depicted in Figure 3. Its main components are:

- MAMES Alert Provider, the network entity responsible for encapsulating the alert messages coming from an alert authority (Alert Issuer) into MAMES message as defined in [17].

- Space Component, represented by a 3-Unit CubeSat (D-SAT), which is in charge of processing and broadcasting the uploaded MAMES Messages.

- Ground Component, which consists of the Satellite Mission Control Center (whose main function is the transmission of the MAMES Messages and the management of the satellite subsystems) and the portable SDR user terminal (used for MAMES messages reception).

As shown in Figure 3, an Alert Protocol Message is sent by the Alert Issuer to the MAMES Alert Provider which encapsulates the received message into a MAMES Message and makes it available (Web Services) for the satellite 


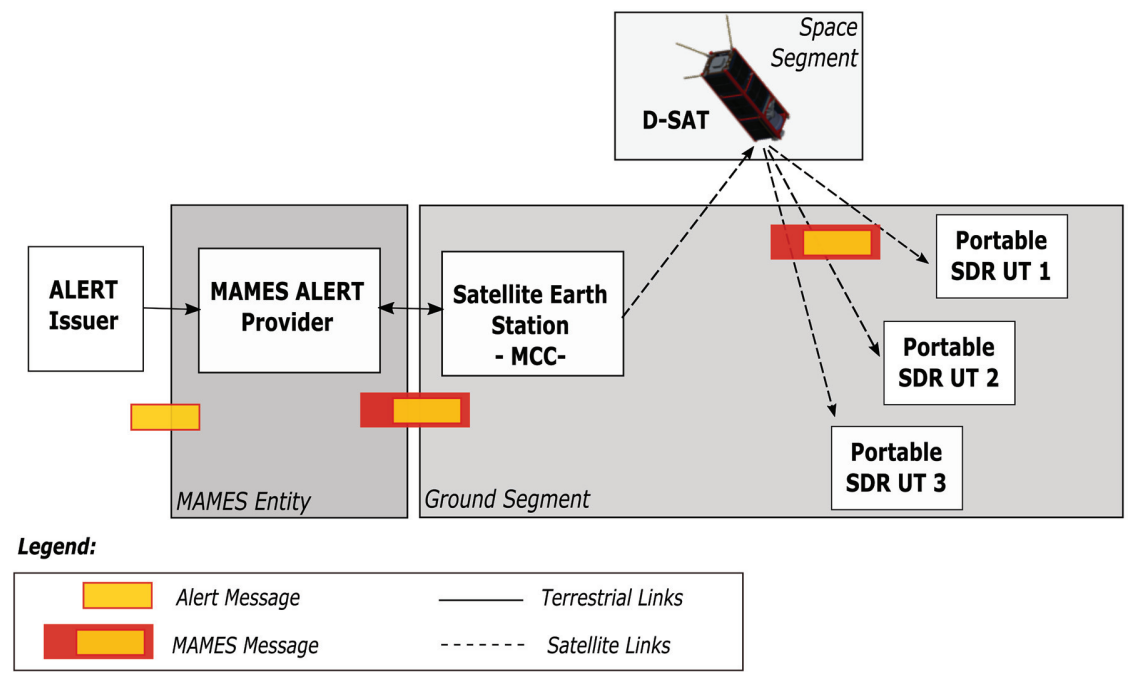

Figure 3 Overall system architecture.

communication network. The Satellite Mission Control Center (MCC) periodically queries the MAMES Alert Provider for updated MAMES Message and uploads it to the D-SAT. After MAMES message on-board processing (validity and priority checks), D-SAT broadcasts the received message back to Earth. The MAMES Message is then received by an SDR receiver.

\subsection{MAMES Protocol}

This section presents the MAMES protocol features relevant for the experimental activity, including the MAMES message structure and the network operations.

The MAMES Protocol $[17,18]$ is defined taking into account the heterogeneity of Alerting Systems and the need of providing a flexible encapsulation scheme for different alert messages (e.g. Common Alert Protocol, unstructured text, image, paging protocols, etc). It defines additional (optional) functions for service extension, enabling the adoption towards a large variety of situations (including network resources limited context). Moreover, it aims to be integrated with the main telecommunication satellite architectures (Galileo Services, DVB-Suite, any IP-based satellite access, etc.) and with already existing terrestrial networks [19]. 


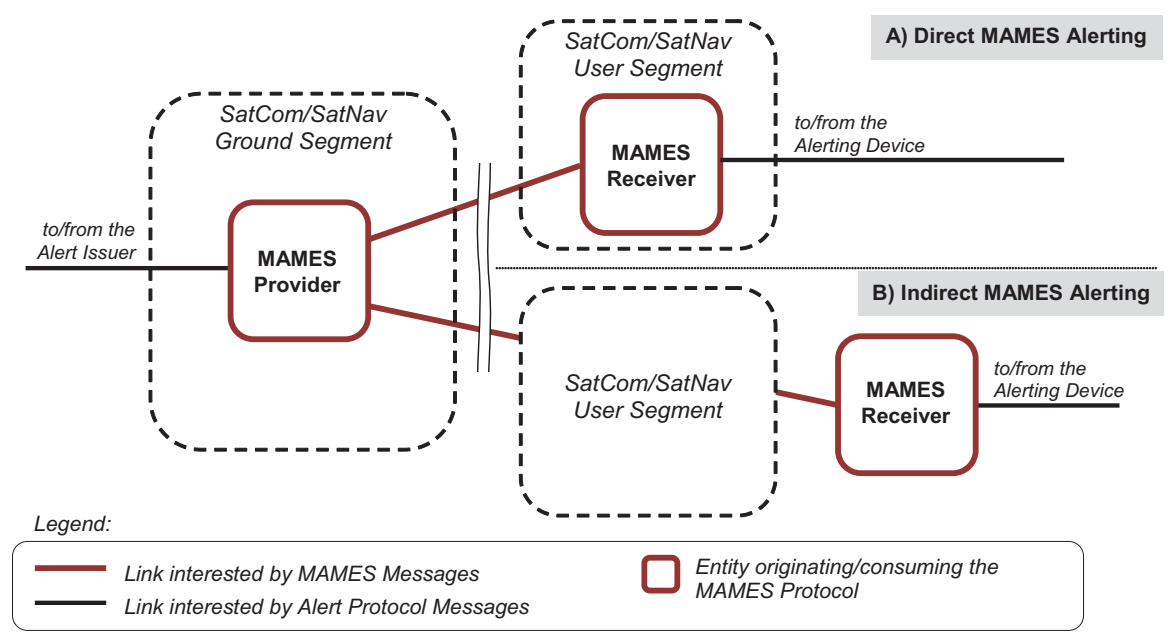

Figure 4 MAMES Operations [18].

One of the main objective of the proposed system is to demonstrate MAMES applicability to LEO satellites (as D-SAT is), showing the functioning of a low complexity alert network, from the generation to the reception of MAMES Messages. The experimental activity focuses on the Direct MAMES Alerting operation mode (Figure 4) [18]: the Portable SDR UT (User Terminal) directly receives MAMES Messages, acting as the satellite terminal and MAMES Receiver (Figure 3).

\subsubsection{MAMES messages design}

Figure 5 reports the extensible structure of the MAMES Frame. It is composed of:

- a set of headers;

- a payload, comprising a concatenation of Alert Protocol Messages (zero, one or multiple Alert Protocol Messages).

About the MAMES Headers, MH (Mandatory Header) and EHs (Extension Headers) pertain to the entire MAMES Message and while the former is of fixed length and contains mandatory information, the latter are optional features (e.g. integrity, encryption, etc.) that enhance the MAMES message. On the other hand, each AMH (Alert Message Header) pertains only to a corresponding Alert Message (AM) contained in the MAMES Payload and specifies the type, language and length of the AM it refers to. 


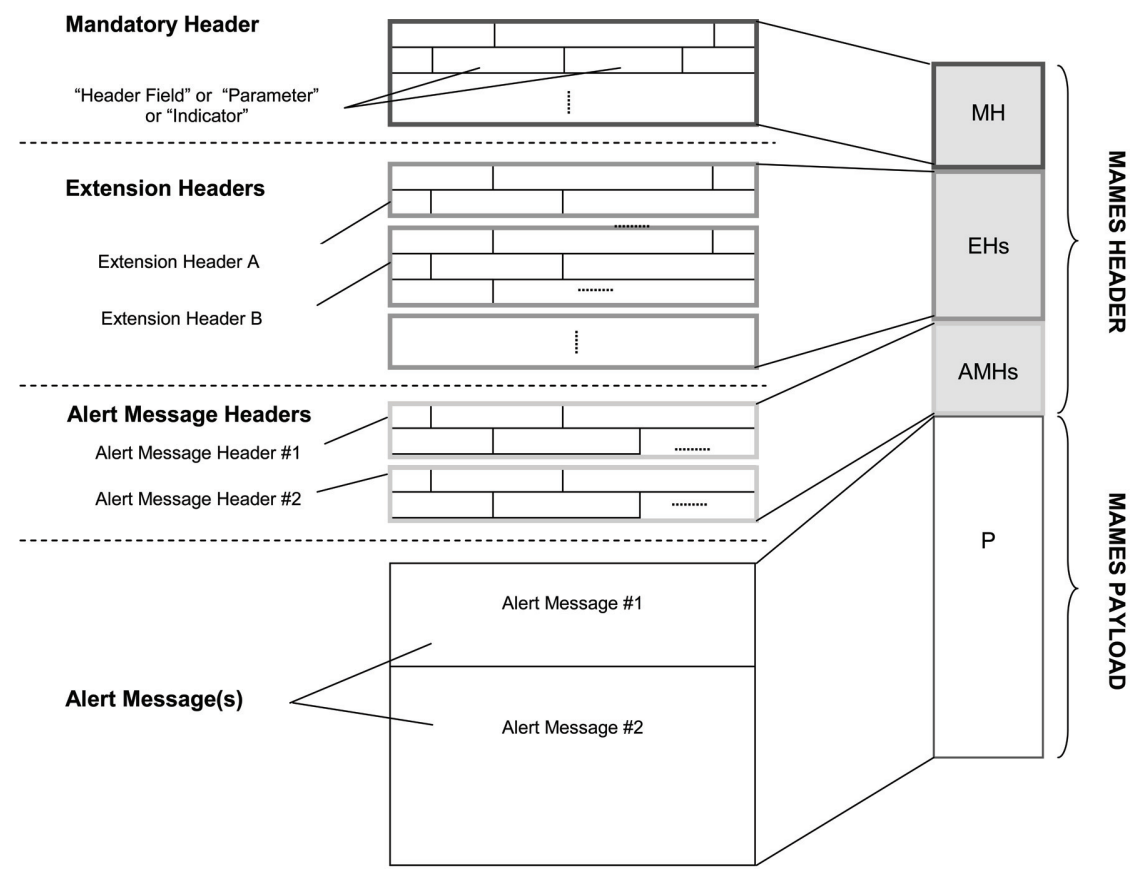

Figure 5 MAMES message structure [18].

Five types of MAMES Messages are defined: i) ALERT, which enables the encapsulation of a single or multiple Alert Protocol Messages; ii) Ultrashort, which represents the shortest MAMES Message and is defined for the transmission of messages over narrowband satellite channels; iii) UPDATE, which contains updated information for a previously transmitted MAMES Message; iv) CANCEL, which declares a previously transmitted MAMES Message as obsolete.; v) ACK, which provides an acknowledgement at MAMES level for a correctly received MAMES Message.

\subsubsection{Experiment relevant MAMES features}

Among the defined MAMES Header fields, it is worth highlighting the role of the priority and time validity information, which are involved in the D-SAT MAMES Message processing, as detailed in the following. The MAMES Frame priority with respect to other MAMES Frames at transport level is managed by the MAMES Transport Priority field of the MH; while the MAMES Validity Start and End fields indicate the time instant when the MAMES Frame shall become valid and invalid, respectively. Upon reception 
of a MAMES Message from the Satellite Earth Station, both the validity and the priority checks (with the previous stored message, if any) are performed on board of the D-SAT. While upon reception of a trigger command for the transmission, the on-board validity verification of the stored message is carried out before the broadcast of the stored message.

\subsection{Space Segment (Platform, COM and SW)}

The platform used for the Alert Network proof-of-concept is a 3-Unit CubeSat. D-SAT is designed according to $3 \mathrm{U}+$ CubeSat standard with a mass lower than $4.5 \mathrm{~kg}$ and a dimension of about $(30 \times 10 \times 10) \mathrm{cm}^{3}$ [20], as illustred in Figure 6 . The satellite since the first conceptual idealization has been designed to maximize the reliability and consequently the mission success. Therefore, a unique feature of D-SAT is that the main functions of the system are single point of failure free [21].
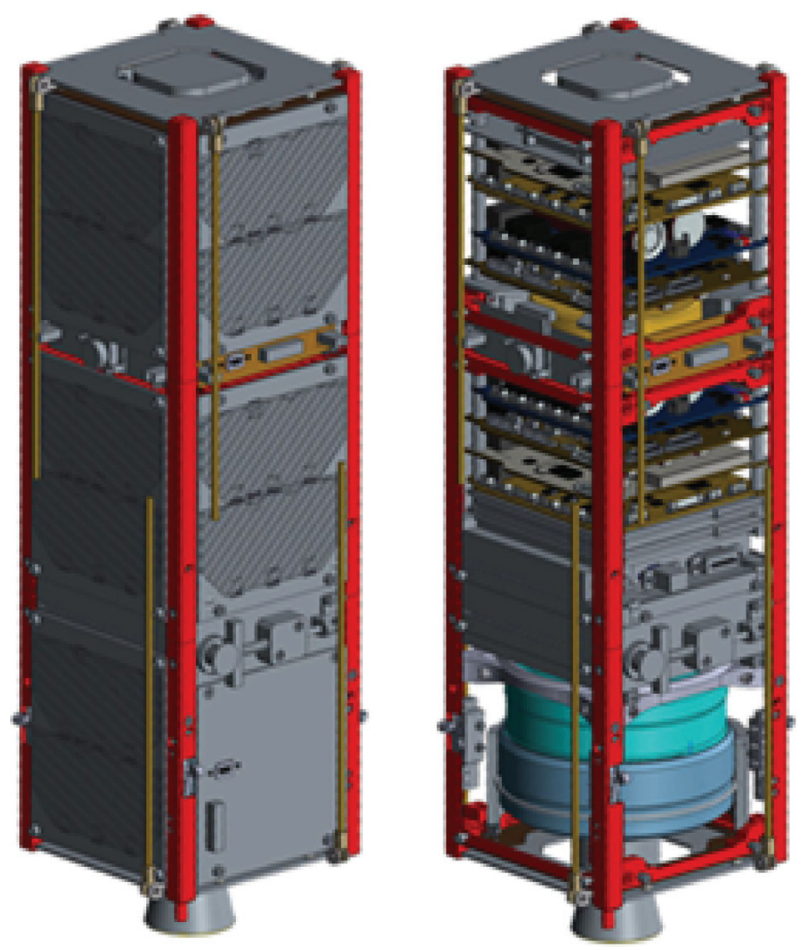

Figure 6 D-SAT render. 


\subsubsection{Platform}

D-SAT spacecraft is constituted by two CubeSat-class systems, called Unit 1 and Unit 2, as shown in Figure 7. Each unit contains the following subsystems:

- an On-Board Computer (OBC) including Attitude and Orbit Control System (AOCS) sensors (magnetometers, sun sensors, gyroscope) and the drivers for AOCS magnetorques;

- a communication subsystem that consists of an UHF radio module and a turnstile antenna;

- an Electric Power System (EPS) including batteries and solar arrays;

- one commanding board for the control of the decommissioning manoeuvre;

- a GPS receiver to increase the accuracy of the Orbit Determination System;

- a spin wheel to impart angular momentum about the z-axis of the satellite, for passive stability during fire;

- an Electro Explosive Subsystem (EES) comprehensive of the EED, the electric "Firing Circuit" and mechanical "Safe and Arm Device" part;

- a solid propellant motor delivering about $750 \mathrm{Ns}$ total impulse to the satellite necessary to directly re-entry from LEO orbit to ground in less than 1 orbit period.

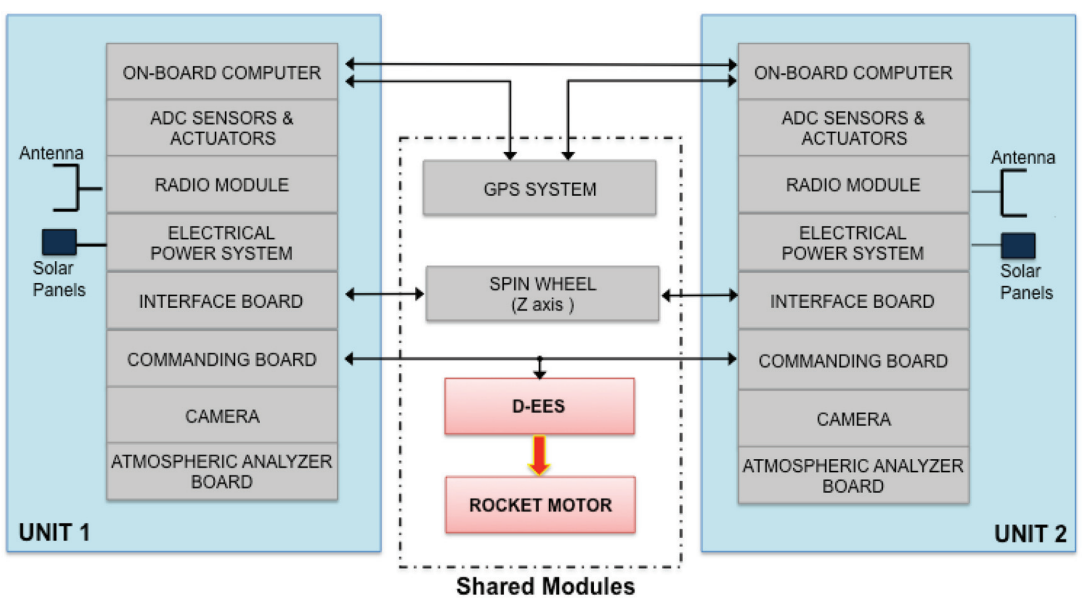

Figure 7 D-SAT block diagram. 
In nominal conditions, Unit 1 is the Master Unit acting as hosting satellite and takes control of the spacecraft, while the Unit 2 acts as "Slave", playing the role of an independent Decommissioning Device and it is set to idle mode. Before the decommissioning phase, the control is transitioned to the Slave Unit, which provides a manoeuvre-suitable attitude, a stabilizing spin motion and finally the deorbiting manoeuvre. Thanks to D-SAT architecture, the decommissioning function is possible even if one of the two units is malfunctioning or dead.

\subsubsection{Communication subsystem}

It is composed by a turnstile omnidirectional antenna and a UHF transceiver module able to transmit up to 1 Watt. The transceiver uses a GMSK modulation over FM signal at a baud rate of 4800 bps: this guarantees a sufficient bandwidth for both telemetry and the service data link. The data are coded with a Reed-Solomon $(223,255)$ coding and randomized according to CCSDS standard [22] to reduce the probability of error and therefore increasing the reliability of the communication link. The UHF communication subsystem has three main functions: i) to transmit a beacon signal (a morse signal FM modulated) as tracking signal; ii) to transmit and receive Telemetry and Tele-command; iii) to transmit and receive MAMES messages.

The link frequency value is $437.505 \mathrm{MHz}$ and it is coordinated by IARU (International Amateur Radio Union) and assigned by ITU. As Transport level, NanoCom U482C uses CubeSat Space Protocol, a small network-layer delivery protocol based on a 32-bit header.

\subsubsection{On-Board software}

The communication subsystem is supported by the satellite on-board computer, which runs a real-time operating system with a high degree of reliability. Among the different functions of the operating system there are the SatAlert Experiment task (SAE) and the Interface SatAlert Experiment task (ISAE). The ISAE manages the software-hardware interaction between the transceiver and the on-board computer guaranteeing the independence of the experiment from the rest of the mission, while SAE implements the logical functions of the MAMES communication protocol. SAE is invoked by the on-board operating system only in the case of an upload of a new MAMES message or a transmission trigger command from the main ground station. When invoked, SAE receives through the ISAE interface information about the uploaded MAMES message, the on-board time and the last MAMES message stored in the transmission queue. Thus, SAE is responsible for MAMES message validation: 
- Time validity check of MAMES message previously stored in the transmission queue, in case of transmission trigger command.

- Time validity and priority checks, in case of an upload of a new MAMES message; SAE parses the received message to compare priority and validity of the new uploaded message with the previously stored message.

Based on the validation results, SAE decides which messages have to be transmitted or deleted from the transmission queue, providing this information to the ISAE interface.

The core of On-Board Computer is a flight proven High-performance 32-bit ARM7 CPU, integrated combined with $2 \mathrm{MB}$ of static RAM, $4 \mathrm{MB}$ of flash memory for data storage and $4 \mathrm{MB}$ of flash memory for code storage. The OBC has an on board timer synchronized with UTC time by means of a GPS receiver within the satellite. The on-board software is also in charge of handling the communication interfaces with other subsystems through data buses (I2C bus, CAN bus and UART).

\subsection{Ground Segment}

\subsubsection{Mission control center}

The D-SAT satellite will be operated by a MCC based in the D-Orbit facility in Fino Mornasco, close to Como lake. The main features of the MCC, which provide a UHF communication link with satellite, are:

- the capability to decode the Morse Beacon Continuous Wave signal and to estimate the frequency of signal that is transmitted by the satellite affected by Doppler effect and temperature shift;

- the possibility to decode and process telemetry and to transmit telecommand to the satellite through the digital GMSK over FM signal;

- the capability of satellite tracking and frequency prediction to compensate the Doppler effect [23].

Almost all the ground station hardware is COTS amateur radio hardware and was selected to meet the requirements imposed by the uplink and downlink budgets (Table 1).

The block diagram of the MCC (Figure 8) includes an equipment that is mounted on the roof-top of the D-Orbit facility and hardware inside the D-Orbit control room.

The antenna section consists of a vertical $70 \mathrm{~cm}$ antenna with linear polarization and a high-gain Yagi with 19 elements that requires accurate pointing. For instance, the $3 \mathrm{~dB}$ half power beam width for the directive is about $21 \mathrm{deg}$ : hence, the ground station includes an azimuth/altitude rotator 
Table 1 Downlink budget table

\begin{tabular}{|c|c|c|c|}
\hline & MCC & SDR UT & \\
\hline Link Budget Parameters & Value & Value & Unit \\
\hline Satellite Range & 909.5 & 909.5 & $\mathrm{~km}$ \\
Elevation Angle & 30 & 30 & $\mathrm{deg}$ \\
Frequency & 437.5 & 437.5 & $\mathrm{MHz}$ \\
Transmission Rate & 4800 & 4800 & $\mathrm{bps}$ \\
\hline Spacecraft & & & \\
Transmitted Power & 1 & 1 & $\mathrm{~W}$ \\
Antenna Gain & 0.4 & 0.4 & $\mathrm{dBi}$ \\
Line Losses & -1.7 & -1.7 & $\mathrm{~dB}$ \\
Pointing Losses & -3 & -3 & $\mathrm{~dB}$ \\
\hline Downlink & $-144,4$ & $-144,4$ & $\mathrm{~dB}$ \\
PathLoss & -3 & 0 & $\mathrm{~dB}$ \\
Polarization Loss & -1.8 & -1.8 & $\mathrm{~dB}$ \\
\hline Atmospheric Losses & & & \\
Ground Station & 17.6 & 5 & $\mathrm{dBi}$ \\
Antenna Gain & 21 & 180 & $\mathrm{deg}$ \\
HBPW & -10.4 & -10.3 & $\mathrm{~dB} / \mathrm{K}$ \\
\hline Figure of Merit, $(\mathrm{G} / \mathrm{T})$ & & & \\
Link Budget Results & -113.5 & -113.5 & $\mathrm{dBm}$ \\
Received Isotropic Power & 28.3 & 18 & $\mathrm{~dB}$ \\
$E_{b} / N_{0}$ & 13.5 & 13.5 & $\mathrm{~dB}$ \\
Required $E_{b} / N_{0} @$ BER $=10^{-5}$ & 14.8 & 4.5 & $\mathrm{~dB}$ \\
Margin $E_{b} / N_{0}$ & & & \\
\hline
\end{tabular}

system which provides a pointing accuracy better than $5 \mathrm{deg}$. Closer to the Yagi antenna is mounted a $20 \mathrm{~dB}$ gain low-noise amplifier (LNA) with a noise figure equal to $0.6 \mathrm{~dB}$ for receiving weak signals. The vertical antenna provides a wider sky coverage and is mainly used to track and acquire D-SAT signal during the Early Phase of the mission when its position is unknown.

The control room contains the remainder of the equipment for the satellite ground station. The transceiver is a Kenwood TS-2000 featuring a high sensitive receiver and high transmit power output. Thanks to a Serial Data interface connected to a computer running Gpredict satellite tracking program, the transceiver frequency can be tuned to compensate for Doppler shift [24]. Gpredict is a real-time satellite tracking and orbit prediction application. It uses the satellite Kepler elements released by NORAD to track D-SAT and computes its position, the time of next passes, the azimuth and elevation angle and the Doppler Shift. 


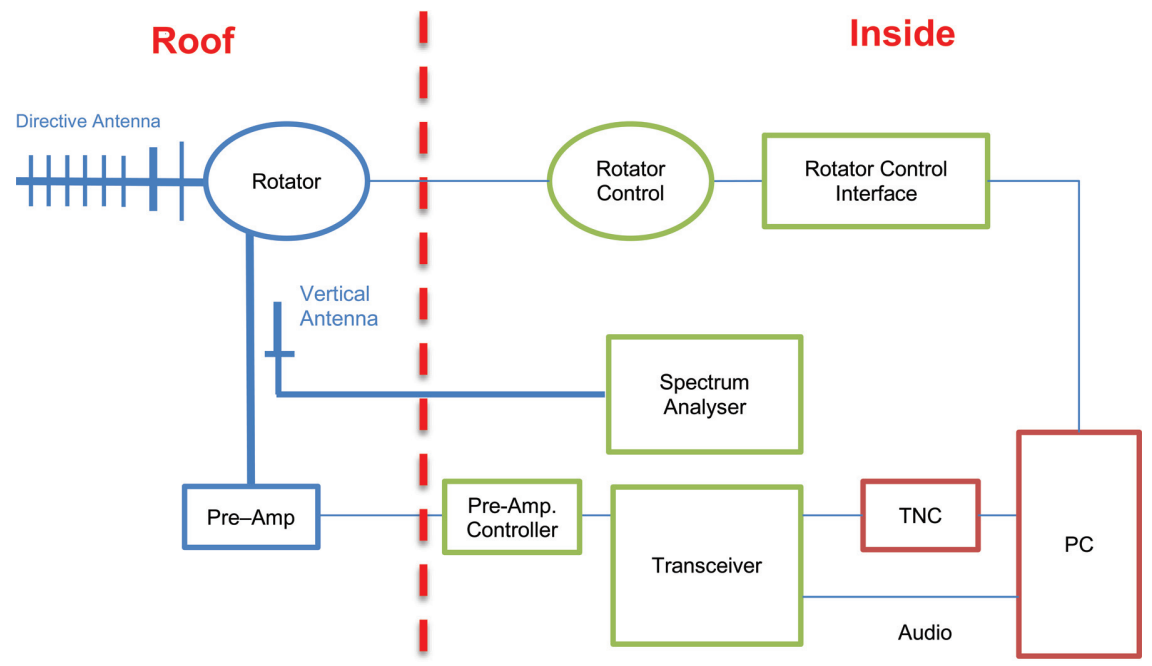

Figure 8 D-SAT MCC block diagram.

The hardware include also a Yaesu G-5500 azimuth and elevator rotators that feed directly into the Rotator Control Port interface with Gpredict. Under this configuration, satellite tracking becomes automated across the sky.

The base baseband modulation-demodulation section, that includes the interface between the computer and the transceiver is delegated to the Terminal Node Controller (TNC). The TNC provides a full-duplex baseband modulation and demodulation using the MSK scheme with baud rates at 4800 baud, a channel coding and decoding function using consisting of a forward errorcorrection (FEC) with an additional scrambling compliant with CCSDS standard and frame format and routing data function according to the CubeSat Space Protocol (CSP) that is based on a simple header structure [25].

\subsubsection{Portable user terminal}

Due to the limited availability of resources and infrastructure characterizing an emergency scenario, a satellite portable user terminal based on an low cost SDR solution that enables a simple and flexible configuration has been designed for the reception of MAMES messages. The SDR Portable UT (Figure 9) includes three main elements: a Circular Polarized Quadrifilar Helix Antenna, an Airspy Mini SDR radio and a personal computer configured with GNU Radio software. The choice of the Quadrifilar Helix Antenna instead of a directive one is justified by the operative scenario and the impossibility of 


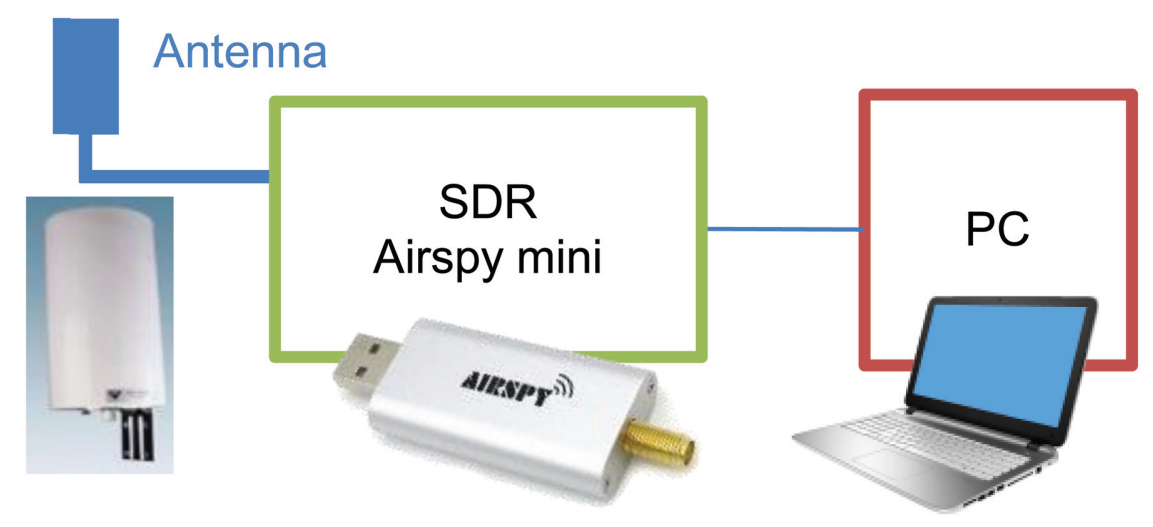

Figure 9 SDR UT block diagram.

using a rotator mechanism to point the satellite. The Helix Antenna ensures a semi-spherical radiation pattern and, although the maximum antenna gain is limited up to $5 \mathrm{~dB}$ the $E_{b} / N_{0}$ value (computed with standard link budget [23]), it is still enough for correctly decoding the signal as shown in Table 1. The used SDR-platform is the AirSpy Mini SDR, supported by a GNU Radio implementation on a computer. The main features of the AirSpy Mini SDR are: a $3.5 \mathrm{~dB}$ Noise Figure in UHF frequency band, a $35 \mathrm{dBm}$ IIP3 RF front end, a 12 bit ADC with a Dynamic Range up to $80 \mathrm{~dB}$ and a $0.5 \mathrm{ppm}$ high precision, low phase noise clock.

The host computer is a Linux system running GNU Radio, an open source radio toolbox with digital signal processing functions and hardware drivers. This is the framework that is used to implement the ground station software. A GNU Radio application usually consists of several modules, called blocks, each performing a specific task. They are connected in a chain, forming a flowgraph, where each block consumes and produces data and the resulting program is a runnable application, referred to as the top block [12]. GNU radio performs all remaining operations of the receiver: modulation and demodulation, error detection and correction, as well as clock synchronization and Doppler compensation [26].

\section{Status and Results}

The D-SAT Qualification Model (QM) has successfully passed the qualification tests campaign following the Launch Service Provider requirements, including mechanical (Sine and Random Vibration, Shock and Acceleration 


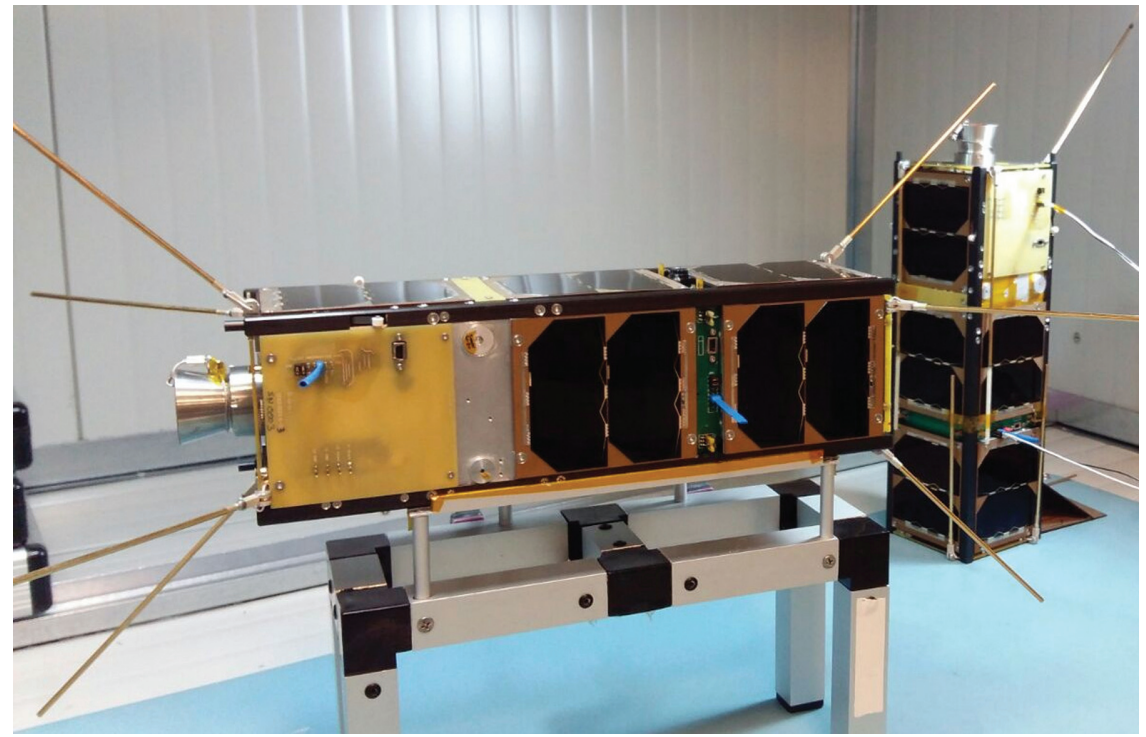

Figure 10 D-Sat FM and QM - courtesy of D-Orbit.

Test) and thermal-vacuum test. The D-SAT Flight Model (FM) integration and acceptance test campaign have been completed. It has been delivered to the Launch Service Provider (LSP), integrated within the P-POD deployer [20] and shipped to the PSLV's launch site in India. The expected launch date is in June 2017. Figure 10 shows both D-SAT models in D-Orbit Clean Room facility.

During functional test activities an extended test campaign of SatAlert operating functions (MAMES Message generation and on-board processing) has been performed with good results. The end-to-end communication between MCC and Portable SDR user terminal was experimented in D-Orbit facility with all the real equipment that will be used during D-SAT mission and emulating the path-loss attenuation using power attenuators in receiving chain.

\section{Conclusions}

An experimental low complexity alert satellite network based on the adoption of a CubeSat, MAMES protocol and SDR implementation has been presented. The experimental activities have proven the feasibility of the proposed alert network and the applicability of MAMES to LEO satellites. 


\section{Acknowledgement}

This study is the result of a cooperation between CNIT (University of Florence) and D-Orbit. The D-SAT project was initially financed by D-Orbit investments and from December 2015 has received funding from the European Union's Horizon 2020 research and innovation programme under grant agreement no. 711193 .

\section{References}

[1] M. Buscher, T. Funke, A. Patzphal, and k. Brie (2016). Satellites might go viral - Data proves it," in Final Proceedings at Small Satellites, System \& Services Symposium (4S), Valletta, Malta.

[2] R. Villain (2016). "Facts \& figures on possible future directions of the small-sat market," in Final Proceedings at Small Satellites, System \& Services Symposium (4S), Valletta, Malta.

[3] N. H. Crisp, K. Smith, and P. Hollingsworth (2015). Launch and deployment of distributed small satellite systems. Acta Astronautica (114), 65-78.

[4] Website: http://www.parabolicarc.com/2016/07/08/report-3600-smallsa ts-clog-space-10-years/

[5] J. A. Larsen and H. P. Mortensen (2013). "In orbit validation of the AAUSAT3 SDR based AIS receiver", in 6th International Conference on Recent Advances in Space Technologies (RAST), Istanbul, pp. 487-491. doi: 10.1109/RAST.2013.6581257

[6] Website: http://www.outernet.is

[7] Website: http://www.astrocast.net/

[8] European Code of Conduct for Space Debris Mitigation - June 2004 Issue 1.0.

[9] S. Antonetti, L. Ferrario, E. Toson and L. Rossettini (2015). "Implementation of Debris Prevention and Reduction Measures by an Independent Propulsive Decommissioning Device," in 66th International Astronautical Congress, Jerusalem.

[10] M. Panizza, C. Sacchi, J. Varela-Miguez, S. Morosi, L. Vettori, S. Digenti, and E. Falletti (2011). "Feasibility study of a SDR-based reconfigurable terminal for emergency applications," in Proceedings of the 2011 IEEE Aerospace Conference, Big Sky, Montana, USA.

[11] T. Ulversy (2012). "Software defined radio on digital communications: A new teaching tool," in 2012 IEEE 13th Annual Conference of Wireless 
and Microwave Technology Conference (WAMICON), vol. 12, p. 18, doi: 978-1-46730128-2

[12] P. Dominguez and Ian (2011). Software Defined Radio: USRP y GNU Radio. Ivn Pinar Domnguez, 1st ed., Vol. 6.

[13] E. Del Re, S. Morosi, S. Jayousi, and C. Sacchi (2009). "SALICE Satellite-Assisted Localization and Communication systems for Emergency services," in Proceedings of the 1st International Conference on Wireless Communications, Vehicular Technology, Information Theory, Aerospace and Electronic Systems (WVITAE), Aalborg, Denmark.

[14] E. Del Re, S. Jayousi, S. Morosi, L.S. Ronga, M. De Sanctis, E. Cianca, M. Ruggieri, E. Falletti, A. Iera, G. Araniti, and C. Sacchi (2013). SALICE project: Satellite-Assisted Localization and Communication Systems for Emergency Services. IEEE Aerospace and Electronics Systems Magazine (99), 4-15.

[15] T. Pecorella, L.S. Ronga, F. Chiti, S. Jayousi, and L. Franck (2015). Emergency satellite communications: research and standardization activities. Communications Magazine (53), 170-177.

[16] D. Iwaizumi, T. Ishida, S. Iino, N. Kohtake, and P. Buist (2014). "GNSS-based emergency message service: Lessons learned and future prospects," in 2014 7th Advanced Satellite Multimedia Systems Conference and the 13th Signal Processing for Space Communications Workshop (ASMS/SPSC), 276-283.

[17] ETSI (2015). "Satellite Earth Stations and Systems (SES); Satellite Emergency Communications; Multiple Alert Message Encapsulation over Satellite (MAMES)", ETSI, TS 103 337, May 2015.

[18] ETSI (2015). "Satellite Earth Stations and Systems (SES); Satellite Emergency Communications (SatEC); Multiple Alert Message Encapsulation over Satellite (MAMES) deployment guidelines ”, ETSI, TR 103 338, May 2015.

[19] L. S. Ronga, S. Jayousi, R. Pucci, S. Morosi, M. Berioli, J. Rammer, A. Fanfani, and S. Antonetti (2015). "Multiple Alert Message Encapsulation Protocol: Standardization and Experimental Activities," in Proceedings of the ISCRAM 2015 Conference, Kristiansand, Palen, Bscher, Comes \& Hughes, eds.

[20] "CubeSat Design Specification" Rev. 13, The CubeSat Program, Cal Poly SLO.

[21] E. Toson, A. Fanfani, S. Morosi, L. S. Ronga, P. Martegani, and L. Rossettini (2016). "D-SAT Mission: An In-Orbit Demonstration of a solid propellant satellite decommissioning device," The 4S Symposium 2016. 
20 A. Fanfani et al.

[22] CCSDS Recommended Standard 131.0-B-2 TM Synchronization and Channel Coding.

[23] G. Maral and M. Bousquet. Satellite Communications Systems: Systems, Techniques and Technology. Pearson Education India.

[24] C. Cooper, R. Fevig, J. Patel. "The CubeSat Ground Station at the University of Arizona".

[25] Technical Datasheet: GS-DS-TNC-4.0 NanoCom TNC1 Datasheet Baseband MSK modem for satellite ground stations.

[26] A. Lofaldli and R. Birkeland (2016). "Implementation of a Software Defined Radio Prototype Ground Station for CubeSats," in Final Proceedings at Small Satellites, System \& Services Symposium (4S), Valletta, Malta.

\section{Biographies}

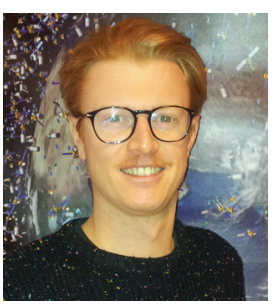

Alessio Fanfani received his Ph.D. degree in telecommunications in 2017 from the University of Florence, Italy. Since 2013, Alessio worked on D-Orbit D-SAT mission as System Engineer and later as Program Manager: he was in charge of the satellite design, integration, verification and testing. He also had the responsibility for design and implementation of a ground station for satellite application operative in VHF/UHF bands. The main research area of interest is Satellite telecommunication services for applications in emergency scenarios. 


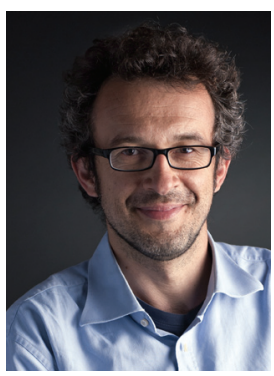

Simone Morosi received his Ph.D. degree in information and telecommunication engineering from the University of Florence in 2000. He is currently an assistant professor at the same university. His present research interests focus on communication and positioning systems, green ICT and energy-efficient wireless communications, and future wireless communication systems. He is an author of more than 120 papers in international journals and conference proceedings.

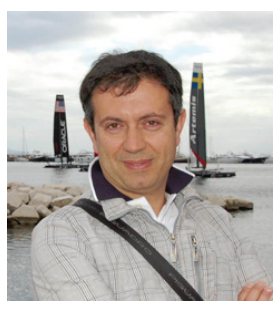

Luca Simone Ronga received his Ph.D. degree in telecommunications in 1998 from the University of Florence, Italy. In 1999 he joined Italian National Consortium for Telecommunications, where he is currently head of research area. His research interests range from signal processing and physical layer to networking and data transport issues and are focused on software defined networking, security for radio communications and satellite systems. He has been principal investigator in several research projects and author of more than 120 papers in international journals and conference proceedings. 


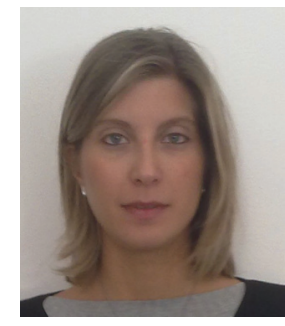

Sara Jayousi received her Ph.D. degree in Computer Science, Multimedia and Telecommunications in 2012 from the University of Florence, Italy. She is with the Department of Information Engineering of the University of Florence since 2008. Her research activity is mainly focused on: satellite communications for emergency, IP QoS network management in hybrid satellite/terrestrial networks, cooperative communications and diversity algorithms in relaying systems. She's author of several transaction and conference papers.

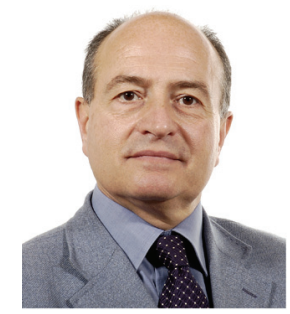

Enrico Del Re has served as full Professor at the University of Florence, Florence, Italy and has been Director of the Department of Information Engineering (DINFO) of the University of Florence and President of the Italian Interuniversity Consortium for Telecommunications (CNIT), having served before as Director. He leaded the Signal Processing and Communications Laboratory of the Department of Information Engineering (DINFO) of the University of Florence. 


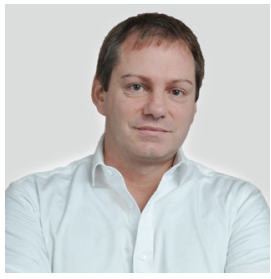

Luca Rossettini is CEO and Founder of D-Orbit, Expert at the Space Advisory Group of the European Commission, Board member of AIPAS (space SME industrial association) and Board member of Confindustria Florence. He is an aerospace engineer with a Ph.D. in advanced space propulsion, awarded with honors by Polytechnic of Milano. He holds a master in Strategic Leadership Towards Sustainability and a Certificate in Technology Entrepreneurship from Santa Clara University, California. 
